\title{
45th Scientific Meeting of the Japanese Society for Mucosal Immunology
}

\section{Contents}

My Meeting Report

Yoshikawa, T. (Kyoto)

Selected Abstracts

Abstracts 1-18

Author Index 


\section{My Meeting Report}

The 45th Scientific Meeting of the Japanese Society for Mucosal Immunology was held at the MielparqueKyoto, July 3-4, 2008. In this meeting, many topics according the immunity of the digestive disease and liver, pancreatic disease were discussed. The membership of this society has been increasing year by year and as of 2008 has surpassed 300 members. Over 170 participants attended the 45th Scientific Meeting, which consisted of one presidential lecture, one special lecture, three symposia and more.

Because of limited space, it is difficult to introduce them all, but I would like to pick out some of the "hot" topics. We hold symposia on "the development of the immunosuppressive therapy for the digestive disease" and "the crosstalk between natural immunity and acquired immunity in the digestive disease". In these symposia, many participants argued eagerly in the field of clinical and basic research on mucosal immunology. Also, we had mini-symposium for "granular leukocyte and the mucosal immunology" that focused on the role of granular leukocyte. In this session, we realized again the importance of granular leukocyte in the intestinal immunity. Especially, the lecture by Prof. Takanori Kanai of Keio University on anti-TNF-alpha therapy against the intestinal inflammation was very interesting and really splendid. Furthermore, the special presentation by Prof. Matthew B. Grisham of LSU Health Science Center on "T-cell Trafficking in the Promotion and Regulation of Chronic Gut Inflammation" gained a lot of attention from the participants. He showed the importance of Tcell trafficking in the development of the intestinal inflammation.

I feel that this meeting is a great opportunity for young specialist in gastrointestinal disease. To share their work on the leading edge of research, I selected the 20 topics in this issue. Finally, I wish to express my deep gratitude to all participants and our colleagues and friends who helped to produce this meeting for their invaluable cooperation and understanding.

Toshikazu Yoshikawa

\footnotetext{
KARGER

Fax +41613061234

E-Mail karger@karger.ch

www.karger.com 


\section{Selected Abstracts}

\section{Digestion}

1

\section{Innate Immunity and Epithelial to Mesenchymal Transition in Biliary Epithelial Cells Are Associated with the Cholangiopathy of Biliary Atresia}

\section{Kenichi Harada, Yasunori Sato, Yasuni Nakanuma \\ Department of Human Pathology, Kanazawa University \\ Graduate School of Medicine, Kanazawa, Japan}

The obliterative lesion of biliary atresia is characterized by a progressive sclerosing cholangitis accompanying severe inflammation, fibrosis, and epithelial injuries. Little is known about the etiology and pathogenesis of biliary atresia, but infections by viruses such as Reoviridae (reovirus and rotavirus) consisting of a double-stranded RNA (dsRNA) have been implicated. Moreover, the epithelial-mesenchymal transition (EMT) has recently been implicated as a mechanism of sclerosing cholangitis in biliary atresia. We hypothesized that the innate immune response to dsRNA in biliary epithelial cells plays an important role in peribiliary fibrosis. Biliary epithelial cells lining in extrahepatic bile ducts and a peribiliary glands in biliary atresia constantly express dsRNA-recognized receptor, TLR3, and frequently showed the activation of NF- $\mathrm{KB}$, a lack of epithelial markers (biliary-type cytokeratin and E-cadherin) and an aberrant expression of mesenchymal marker (intermediate filament vimentin). In vitro study using cultured human biliary epithelial cells revealed that the stimulation with poly(I:C) (a synthetic analog of viral dsRNA) up-regulated the expression of basic fibroblast growth factor (bFGF) and down-regulated those of Bambi (Transforming growth factor- $\beta 1$ (TGF- $\beta 1$ ) pseudoreceptor) and the epithelial markers. Each bFGF and TGF- $\beta 1$ also decreased the epithelial markers and increased vimentin. These suggest that EMT of biliary epithelial cells is associated the histogenesis of sclerosing cholangiopathy in biliary atresia and that biliary innate immunity to dsRNA viruses induces biliary epithelial cells to undergo EMT via the production of bFGF and the increased susceptibility to TGF- $\beta 1$.
2

The Possible Involvement of IL-8 Production via Protease-Activated Receptors (PAR) in H.Pylori-Associated Gastritis

Ikuhiro Hirata, Norimasa Yoshida, Katsura Mizushima, Tatsushi Omatsu, Hirokazu Kajikawa, Tomohisa Takagi, Osamu Handa, Satoshi Kokura, Yuji Naito, Toshikazu Yoshikawa

Gastroenterology and Hepatology, Kyoto Prefectural University of Medicine, Kyoto, Japan

Background and Aims: Some serine proteases play important roles in the inflammatory and immunological reaction by generating proinflammatory cytokines via protease-activated receptors (PARs). Furthermore, it has been shown that PARs are involved in cell proliferation and apoptosis. Recently, we have reported that H.pylori induces the expression of PAR1 and PAR2 on the cultured gastric epitherial cells, and that some proteases derived from H.pylori promote IL-8 production via PARs. In this study, we revealed the relationship among the expression of PAR, IL-8 production and H.pylori infection using biopsy samples from human gastric mucosa.

Materials and Methods: After informed consent was obtained, we took biopsy specimens of gastric(antrum, body) mucosa from 21 patients with H.pylori-infection and 14 patients without H.pyloriinfection. The diagnosis of H.pylori infection was performed by the rapid urease test or microscopy. We studied the expression of PAR1, PAR2 and IL-8 in H.pylori-positive/negative gastric mucosa by realtime PCR and immunohistochemistry. Moreover, we evaluated the difference in these expression after H.pylori eradication.

Results: Constitutive expression of PAR1 and PAR2 was observed in the H.pylori-negative gastric mucosa. Expression level of PAR1, PAR2 and IL-8 was significantly higher in the H.pyloripositive gastric mucosa than that of H.pylori-negative. It showed correlation between the expression level of PAR and IL-8. The expression of PAR1, PAR2 and IL-8 was decreased after H.pylori eradication.

Conclusions: These results indicate that H.pylori infection increases in PARs expression which is partially involved in IL-8 production, suggest that PARs are implicated in the pathogenesis of H.pylori -associated gastritis.

\begin{tabular}{ll}
\hline KARGER & (c) 2008 S. Karger AG, Basel \\
Fax +4161306 1234 & 0012-2823/08/0783-0105\$24.50/0 \\
$\begin{array}{l}\text { E-Mail karger@karger.ch } \\
\text { www.karger.com }\end{array}$ & $\begin{array}{l}\text { Accessible online at: } \\
\text { www.karger.com/dig }\end{array}$
\end{tabular}




\section{3}

\section{Role of Ghrelin and Its Receptor in Inflammatory Bowel Disease}

Shuhei Hosomi, Nobuhide Oshitani, Naoto Hirata, Mizuki Kono, Takehisa Suekane, Noriko Kamata, Mitsue Sogawa, Hirokazu Yamagami, Kenji Watanabe, Kazunari Tominaga, Toshio Watanabe, Yasuhiro Fujiwara, Tetsuo Arakawa

Department of Gastroenterology, Osaka City University Graduate School of Medicine, Osaka, Japan

Background: Ghrelin, a novel endogenous ligand for the growth hormone secretagogue receptor (GHSR), has been demonstrated to possess multiple functions including anti-inflammatory effects. Although ghrelin has recently been found to play important roles in the immune system, the roles of ghrelin and GHSR in IBD are little understood. The aim of this study was to investigate the expression of ghrelin and GHSR and the function of ghrelin in IBD.

Methods: The expression of ghrelin and GHSR mRNA was quantified in mucosal biopsy specimens from controls, patients with $\mathrm{CD}$, and patients with UC using quantitative RT-PCR. The locations of ghrelin and GHSR were investigated immunohistochemically in surgically resected specimens. We also evaluated the percentage of GHSR-positive PBMCs in healthy controls and patients with CD by flow cytometry.

Results: Ghrelin mRNA levels in colonic mucosa of IBD were higher than control level. GHSR-1a mRNA level in active CD was significantly higher than control level. Ghrelin and GHSR-1a were expressed on CD3- and CD68-positive cells. The percentage of GHSR-1a-positive cells among $\mathrm{T}$ cells in the lamina propria was significantly higher in the patients with $\mathrm{CD}$ than in the controls. The percentage of GHSR-1a-positive peripheral blood T cells in patients with $\mathrm{CD}$ was also significantly higher than control level. Stimulation of human T cells with ghrelin increased expression of Th-2 cytokines.

Conclusions: Our findings demonstrate that ghrelin may play an important role in the immune system in CD.

\section{4}

Role of Aryl Hydrocarbon Receptor (AhR) for Epstein-Barr Virus Reactivation of Pathogenesis in Sjogren's Syndrome

Hiroko Inoue, Sachi Yamamoto, Kumi Obara, Kenji Mishima, Ichiro Saito

Department of Pathology, Tsurumi University School of Dental Medicine, Yokohama, Japan

Epstein-Barr virus (EBV) reactivation is thought as a causative factor for gastric carcinoma, infectious mononucleosis and autoimmune diseases including Sjogren's syndrome (SS). However, the physiological stimuli responsible for the EBV activation in the patients have not been characterized. 2,3,7,8-tetracholorodibenzo(p) dioxin, (TCDD) and certain polycyclic aromatic hydrocarbons are widespread environmental pollutants that are designated human carinogenes, immunomodulator and virus activator. In this study, we used the firefly luciferase plasmids connected with promoters of BZLF1, indispensable and sufficient for viral reactivation, and CYP1A1, target gene for aryl hydrocarbon receptor (AhR). We have found that xenobiotics bound AhR upregulated the BZLF1 promoter activity in salivary epithelial cells, HSY. This result suggested that dioxins might activate EBV reactivation in epithelial cells. Next, to confirm the effect of dioxins for the pathogenesis of SS, we analyzed promoter activity of CYP1A1 in saliva from SS $(n=10)$ and control individuals $(\mathrm{n}=10)$. The average CYP1A1 activity induced by SS saliva (3.56-fold) was higher than by control saliva (1.61fold). Moreover, a significant correlation was observed between the level of CYP1A1 and BZLF1 promoter activities, and the amount of EBV DNA in each saliva sample of SS patients was parallel to the BZLF1 promoter activity. These results suggest that xenobiotics activated AhR in SS saliva might enhance the EBV reactivation in salivary epithelial cells, and might have causative effect on EBV related diseases.

5

\section{The Efficacy of Hyperthermia Combined with Cancer Immunotherapy}

Satoshi Kokura, Tetsuya Okayama, Takeshi Ishikawa, Satoko Adachi, Tomohisa Takagi, Osamu Handa, Yuji Naito, Toshikazu Yoshikawa

Molecular Gastroenterology and Hepatology, Kyoto Prefectural University of Medicine, Kyoto, Japan

Aim: Several lines of evidence from recent years support the immunomodulation by hyperthermia, especially studies of HSPs as a source of antigen due to their ability to chaperone intracellular peptides and as a maturation signal for dendritic cell. In this study, we evaluated the cytotoxicity of human peripheral mononuclear cells (PBMC), tumor infiltrating lymphocyte (TIL), and lymphokine activated killer (LAK) against for autologous cancer cell or K-562 after heat treatment, subjecting the target and effector cell to heat treatment.

Methods: TIL obtained from pleural effusion or acsites of cancer patient, anti-CD3 and lymphokine activated killer cell (LAK), and PBMC from healthy volunteers were used as effector cells. These effector cells were subjected heat treatment at the temperature of $37^{\circ} \mathrm{C}$ or $43^{\circ} \mathrm{C}$ for $1 \mathrm{~h}$. On the other hand, K-562 and cancer cell obtained from acsites or pleural effusion of patients with solid cancer were used as target cells. These target cells were subjected heat treatment at the temperature of $37^{\circ} \mathrm{C}$ or $43^{\circ} \mathrm{C}$ for $1 \mathrm{~h}$. We co-cultured effector cells with heat treatment and target cells without heat treatment, or effector cells without heat treatment and target cells with heat treatment at $37^{\circ} \mathrm{C}$ for $4 \mathrm{~h}$ and evaluated cytotoxicity of 3 kinds of effector cells. In addition, we measured NK cells activity in PBMC from healthy volunteers and cancer patient before and after hyperthermia.

Results: (1) The cytotoxicity of PBMC against for K-562 was down-regulated immediately after heat treatment, but recovered and got over baseline at $24 \mathrm{~h}$ after. The cytotoxicity of LAK got weak till $3 \mathrm{~h}$ after heat treatment. (2) Heat treatment against for cancer cell enhanced the cytotoxicity of TIL but showed no effect for PBMC and LAK. (3) The NK cells activity of PBMC from healthy volunteers was down-regulated immediately after heat treatment but recovered 
and got over baseline at $24 \mathrm{~h}$ after, however that from cancer patient didn't get over baseline till $48 \mathrm{hr}$ after.

Conclusion: We conclude that combination of hyperthermia and cancer immunotherapy may be effective, and patients shouldn't be treated with hyperthermia immediately after administration of LAK.

\section{6 \\ Effect of Psychological Stress on Intestinal Inflammation}

Hisayuki Matsunaga, Ryota Hokari, Chie Kurihara, Koichi

Takebayashi, Keisuke Okudaira, Chikako Watanabe,

Shunsuke Komoto, Mitsuyasu Nakamura, Yoshikiyo Okada,

Atsushi Kawaguchi, Shigeaki Nagao, Yoshikazu Tsuzuki,

Soichiro Miura

Department of Internal Medicine, National Defense

Medical College, Saitama, Japan

Background and Aims: The pathogenesis of inflammatory bowel disease (IBD) such as ulcerative colitis and Crohn's disease is still unknown. The pathogenesis has a complex, multifocal etiology comprising genetic and environmental factors, which are associated with dysregulation of the mucosal immune system. However, the role of stress in the pathogenesis of IBD is controversial. In animal studies exposure to various stressors, including restraint stress or a combination of restraint and cold stress were used. But it is difficult to differentiate the influence of psychological stress from that of physical stress by these previous methods. In this study we investigated the effect of psychological stress without physiological stress on colonic inflammation and the change of cytokine expression in mice using communication box paradigm.

Materials and Methods: Eight weeks aged C57BL/6 mice were used for this study. Mice were administered by dextran sodium sulfate dissolved in water for 5 days and then were taken socio-psychological stress in communication box. Body weight and colonic length were measured and the colon was stained by hematoxylin and eosin (H.E.). The expressions of pro-inflammatory cytokines and adhesion molecules in the colon were analyzed by RT-PCR.

Results: DSS treatment significantly decreased body weight and colonic length compared with control and it was confirmed to induce colitis by H.E. staining. Addition of psychological stress exacerbated colonic inflammation further. The addition of stress increased the expression levels of pro-inflammatory cytokines and adhesion molecules in the colon.

Conclusions: We demonstrated that psychological stress without physiological stress exacerbated colonic inflammation in mice.
7

\section{Analysis of Regulatory $\mathbf{T}$ Cells in Patients with Autoimmune Pancreatitis}

\author{
Hideaki Miyoshi', Kazushige Uchida' ${ }^{1}$, Norimasa Fukata ${ }^{1}$ \\ Masanori Koyabu', Takao Taniguchi², Syuziro Yazumi', \\ Mitsunobu Matsushita', Makoto Takaoka', Kazuichi \\ Okazaki \\ ${ }^{1}$ The Third Department of Internal Medicine, \\ Kansai Medical University, ${ }^{2}$ Department of Internal \\ Medicine, Otsu Red Cross Hospital, ${ }^{3}$ Department of \\ Gastroenterology and Hepatology, Kyoto University \\ Graduate School of Medicine, Japan
}

Background and Aim: Autoimmune pancreatitis (AIP) is a new clinical entity of pancreatic disorder. Typical immunological abnormalities are increased levels of serum gammaglobulin, IgG4, and the presence of autoantibodies. The histopahtological findings show lymphoplasmacytic sclerosing pancreatitis, which are fibrotic changes with dense infiltration of lymphocytes and IgG4-positive plasmacytes. Recently, regulatory $\mathrm{T}$ cells (Tregs) have been reported to be involved in the development of various autoimmune diseases as well as B-cell shifting to IgG4 producing plasmacytes. To clarify the role of CD4+CD25 high Tregs in pathophysiology of AIP, we analyzed Tregs in patients with autoimmune pancreatitis.

Subjects and Methods: We recruited 37 patients with AIP for this study ( 21 untreated patients and 16 patients treated with corticosteroids). For comparison, we also recruited 41 patients with other pancreatic disease (21 patients with alcoholic pancreatitis and 20 patients with idiopathic pancreatitis) and 39 healthy subjects. We analyzed Tregs as CD4+CD25high, CD4+CD25+CD45RA+ (naïve) and CD4+CD25+IL-10+cells from peripheral blood by flow cytometry.

Results: In peripheral blood, CD4+CD25high Tregs were significantly increased in AIP patients compared to alcoholic chronic pancreatitis $(\mathrm{CP})$, idiopathic $\mathrm{CP}$, and healthy control $(\mathrm{P}<0.05)$. Naïve (CD4+CD25+CD45RA+) Tregs significantly decreased in AIP compared to healthy control, alcoholic $\mathrm{CP}$, and idiopathic $\mathrm{CP}(\mathrm{P}<0.05)$. In untreated AIP patients, the number of CD4+CD25high Tregs and IgG4 are positively correlated $(\mathrm{R}=0.50)$. The number of IL-10 producing Tregs and IgG4 are also positively correlated $(\mathrm{R}=0.53)$.

Conclusion: Increased numbers of CD4+CD25high Tregs and IL-10 produced by the cells may influence IgG4 production in AIP, while decreased numbers of naïve Tregs may be involved in the pathogenesis of AIP. 


\section{8}

\section{Thioredoxin-Binding Protein-2 Deficiency in Mice Suppresses Methionine-Choline- Deficient Diet-Induced Non-Alcoholic Steatohepatitis}

\author{
Hajime Nakamura1, Md. Kaimul Ahsan¹, Hiroaki Okuyama1, \\ Hiroshi Masutani², Junji Yodoi \\ ${ }^{1}$ Thioredoxin Project, Department of Experimental \\ Therapeutics, Translational Research Center, Kyoto \\ University Hospital, ${ }^{2}$ Laboratory of Infection and \\ Prevention, Department of Biological Responses, Institute \\ for Virus Research, Kyoto University, Kyoto, Japan
}

In non-alcoholic fatty liver disease, oxidative stress is believed to play a crucial role as a second-hit for the progression of simple steatosis to steatohepatitis. Thioredoxin (TRX) is a potent antioxidant molecule including anti-apoptotic and anti-inflammatory functions. TRX-binding protein-2 (TBP-2) is an endogenous negative regulator of TRX. Deficiency of TBP-2 in mice causes lipid dysfunction such as hyperlipidemia and hepatic steatosis in glucose-deficient state. The aim of this study was to investigate the role of TBP-2 in the development of non-alcoholic steatohepatitis (NASH). TBP-2-deficient (TBP-2 ${ }^{-/}$) and wild type (WT) mice were fed either a normal or methionine-cholinedeficient (MCD) diet for up to 10 weeks. Pathophysiological state of the liver was evaluated by means of biochemical and immunohistochemical analyses. Compared with WT mice, TBP- $2^{-/-}$mice showed severe simple steatosis rather than steatohepatitis. However, oxidative stress determined by lipid peroxidation and DNA damage, neutrophil-infiltration and fibrosis were attenuated in TBP- $2^{-/-}$mice. PCR analysis showed the expressions of inflammatory-cytokine-related genes and fibrosis-inducing genes were less in TBP- $2^{-/-}$mice. These results suggested that oxidative stress was attenuated in TBP- $2^{-/-}$mice by the relatively increased function of TRX or by the dysfunction in lipid metabolism. TBP-2 might play a crucial role in the pathogenesis of NASH.

\section{9 \\ Role of Interleukin-17 in Helicobacter Pylori- Induced Gastritis}

Koji Otani, Toshio Watanabe, Tetsuya Tanigawa, Hirotoshi Okazaki, Hirokazu Yamagami, Kenji Watanabe, Kazunari

Tominaga, Yasuhiro Fujiwara, Nobuhide Oshitani, Tetsuo Arakawa

Department of Gastroenterology, Osaka City University Graduate School of Medicine, Japan

Background: Helicobacter pylori (H. pylori)-induced immune responses are skewed toward a T helper (Th) 1 phenotype, indicated by a predominance of interferon (IFN)- $\gamma$ and tumor necrosis factor (TNF)- $\alpha$. A novel and unique subset of interleukin (IL)-17-producing Th17 cells has recently been discovered. IL-12, IL-4, and IL-23 promote Th1, Th2, and Th17 cell differentiation, respectively.

Aim: To examine the expression and role of IL-17 in H. pyloriinduced gastritis in mice.
Methods: Mice were inoculated with $H$. pylori (SS 1 strain). Six months later, the mice were subjected to intraperitoneal injection of rat monoclonal anti-IL-17 neutralizing antibody $(100 \mu \mathrm{g} /$ day $)$ or rat irrelevant IgG2a (100 $\mu \mathrm{g} /$ day $)$ once a day for 3 days. Gastric tissue was subjected to measurement of myeloperoxidase (MPO) activity, assay of levels of mRNAs for cytokines by real-time RT-PCR, and immunofluorescence study.

Results: H. pylori infection caused a 24 -fold increase in IL-17 mRNA expression. Double-immunofluorescence examination showed that IL-17 proteins were mainly expressed on $\mathrm{CD}^{+} \mathrm{T}$ cells, macrophages and dendritic cells. H. pylori infection also elevated mRNA levels for IL-12, IFN- $\gamma$, and TNF- $\alpha$ (Th1-type inflammatory cytokines) by 17.2-fold, 93.2-fold, and 10.2-fold, respectively, with a 9.8-fold increase in MPO activity, while it did not affect mRNA levels for IL-4 and IL-5 (Th2-type inflammatory cytokines). Compared to $H$. pylori-infected controls given irrelevant IgG2a, treatment with anti-IL-17 antibody elevated mRNA levels for IFN- $\gamma$ and TNF- $\alpha$ by 2.6-fold, and 1.6-fold, respectively, and also increased MPO activity by 2.1 -fold, while it did not affect expression of IL-12 mRNA.

Conclusion: These findings suggest that IL-17 exerts antiinflammatory effects on $H$. pylori-induced gastritis via suppression of Th1 cell differentiation.

10

\section{The Balance of Peripheral Blood and Intrahepatic Treg and Th17+ Cells may Contribute to the Pathogenesis of Chronic Hepatitis $\mathbf{C}$ and Autoimmune Hepatitis}

Keisuke Ojiro, Hirotoshi Ebinuma, Kanji Wakabayashi, Yoshiyuki Yamagishi, Shinichiro Tada, Hidetsugu Saito, Toshifumi Hibi

Division of Gastroenterology and Hepatology, Department of Internal Medicine, Keio University, Japan

Effector T-cell response is impaired in patients with persistent infection of $\mathrm{HCV}$, in which increase of CD4+CD25+Foxp3+ Treg is a possible pathogenesis of viral persistence. A distinct CD4+ lineage, Th17, have been implicated to associate with autoimmune or infectious diseases, and the differentiation of Treg and Th17 is regulated by same cytokines such as TGF- $\beta$ and IL-6. We aimed to investigate the frequency of Treg and Th17 in both peripheral blood mononuclear cells (PBMC) and intrahepatic cells in patients with $\mathrm{HCV}$ infection and autoimmune hepatitis. The frequency of CD4+FOXP3+ cells and CD4+IL-17+ cells in PBMC were examined by flowcytometry in patients with untreated chronic hepatitis $\mathrm{C}(\mathrm{CH}-\mathrm{C}, \mathrm{n}=16)$, liver cirrhosis type $\mathrm{C}(\mathrm{LC}-\mathrm{C}, \mathrm{n}=15)$ and $\mathrm{AIH}$ $(n=6)$. Intrahepatic FOXP3+ cells and IL-17+ cells were also examined by immunohistochemistry in the needle biopsy samples from patients with $\mathrm{AIH}(\mathrm{n}=26)$. Clinical characteristics of the patients were analyzed in comparison to the results of flocytometry and immunohistochemistry. The frequency of peripheral CD4+FOXP3+ cells from patients with $\mathrm{CH}-\mathrm{C}$ and LC-C was higher than those from healthy control, although the difference was not significant. The ratio of IL-17+/FOXP3+ tended to be low in these patients. In patients with AIH, the frequency of intrahepatic IL-17+ cells was lower in patients with acute-onset type than those with chronic type. The intrahepatic ratio of IL-17+/FOXP3+ was higher in acute-onset group than in chronic group. These results suggest 
that the balance of Treg and Th17+ cells is implicated in the persistent infection of the virus and severity of autoimmune reaction.

11

\section{The Study about the Efficacy for Advanced Digestive Cancers with Hyperthermia and Immunotherapy}

\author{
Tetsuya Okayama', Satoshi Kokura², Jun Funaki', Tsuyoshi \\ Ishikawa², Tomohisa Takagi², Osamu Handa², Yuji Naito², \\ Kazuki Nakane ${ }^{3}$, Takahisa Takeda ${ }^{3}$, Tadao Ohno ${ }^{4}$, Toshikazu \\ Yoshikawa ${ }^{2}$ \\ ${ }^{1}$ Inflammation and Immunology, ${ }^{2}$ Molecular \\ Gastroenterology and Hepatology, Kyoto Prefectural \\ University of Medicine, ${ }^{3}$ Takeda immunotherapy Clinic, \\ ${ }^{4}$ Cell-Medicine, Inc., Japan
}

Aim: We give advanced cancer patients medical treatment with hyperthermia and immunotherapy. In this study, we discuss the efficacy for advanced cancer patients of immunotherapy.

Methods: For immunotherapy, we used vaccine therapy such as autologous formalin-fixed tumor vaccine and cellular immunotherapy such as CD3-LAK and TIL therapy. We do vaccine therapy for 49 patients, and cellular immunotherapy for 75 patients as CD3-LAK, and 5 patients as TIL therapy.

For the adjudication of therapeutic effect, we check the cumulative survival rate, and for the adjudication of immuno-response, we check the variation of cytokike, the classification of PBMC, the NK activity, and LAK activity.

Results: For the patients treated with immunotherapy, we admitted the prolongation of cumulative survival rate, and the improvement of the immunological index.

Conclusions: We get the degree of the efficacy for the advanced cancer patients treated with immunotherapy. On the contrary, we don't get the clinical efficacy despite the improvement of various immunological index. In this mechanism, it is suggested the involvement of cancer immuno-escape mechanism, we plan to do further examinations for this mechanism.

\section{2}

\section{Role of Autoantibodies in the Experimental Autoimmune Gastritis: Prevention by Anti-FcR Antibodies}

\author{
Saito T. ${ }^{1}$, Sun $X^{2}{ }^{2}$, Suzuki $K^{2}{ }^{2}$, Kushida Y. ${ }^{1}$, Kawauchi $Y^{2}$, \\ Asakura H. ${ }^{2}$, Nagafuchi S. ${ }^{3}$, Hosono M. ${ }^{1}$ \\ ${ }^{1}$ Dept. Life Science, Grad. School Sci. and Technol., ${ }^{2}$ Dept. \\ Gastroenterology and Hepatology, Grad. School Med \\ and Dent Sci., Niigata University, ${ }^{3}$ Dept. Health Science, \\ School of Medicine, Kyushu University, Japan
}

Aim: Autoimmune gastritis (AIG) in neonatally thymectomyzed mice is thought to be a good model for human AIG with pernicious anemia. Since the pathological meanings of these antibodies that generally appear in AIG mice are obscure, exploring their role is crucial for understanding the mechanism of AIG generation and extension.

Methods: Neonatally thymectomized B-cell deficient ( $\left.\mathrm{B}^{-}\right)$mice with a mutation at the immunoglobulin $\mu$ chain gene received an extrinsic supplement of autoantibodies, and neonatally thymecomized wild type $\left(\mathrm{B}^{+}\right)$mice were used to investigate the effect of the treatment with anti-FcR antibodies on AIG development.

Results: 1) Neonatal thymectomy induced AIG in a given proportion of the $\mathrm{B}^{+}$, but not in the $\mathrm{B}^{-}$mice. 2) In the $\mathrm{B}^{-}$mice, AIG was developed as the same extension as AIG in wild type animals, when B cells were reconstituted by an injection of the wild type's bone marrow cells. 3) When they received autoantibodies once during the weaning period, AIG was even generated in the $\mathrm{B}^{-}$mice. 4) When treatment with autoantibodies was applied to wild types of mice, AIG pathogenesis extended more severely without changes in AIG incidence. 5) Speculating that autoantibodies may be engaged in the antigen-concentration and/or a given cell's activation mediated through the function of the antibodies' Fc portion, treatment with anti-FcR (CD16/32) antibodies did significantly reduce AIG incidence and its pathological severity.

Conclusion: Through the FcR-mediated event, autoantibodies appearing in AIG promote the development and severity that was prevented by an injection of anti-FcR antibodies.

\section{3}

\section{Interleukin-32 $\alpha$ Expression in Inflammatory Bowel Disease}

Makoto Shioya, Atsushi Nishida, Yuhki Yagi, Osamu Inatomi, Atsuhiro Ogawa, Tomoyuki Tsujikawa, Akira

Andoh, Yoshihide Fujiyama

Department of Medicine, Shiga University of Medical Science, Japan

Background and Aim: Interleukin (IL)-32 is a recently described proinflammatory cytokine, characterized by induction of TNF- $\alpha$. We studied IL-32 $\alpha$ expression in the inflamed mucosa of inflammatory bowel disease (IBD). We also investigated mechanisms regulating IL-32 $\alpha$ expression.

Methods: Tissue samples were obtained endoscopically or surgically from patients with ulcerative colitis $(\mathrm{UC})(\mathrm{n}=5)$, Crohn's disease (CD) $(n=8)$ and normal colorectal tissues $(n=5)$. IL-32 $\alpha$ expression was evaluated by standard immunohistochemical procedure. To investigate the mechanisms of IL-32 induction, we used epithelial cell line, HT-29,T-84 and Caco-2. IL-32 mRNA expression was analyzed by real time-polymerase chain reaction and Northern blot.

Results: IL-32 $\alpha$ was weakly expressed by intestinal epithelial cells from normal individuals. In the inflamed mucosa of IBD patients, epithelial IL-32 $\alpha$ expression was markedly increased. IL-32 $\alpha$ mRNA expression was significantly increased in samples from UC and CD patients as compared to those from normal individuals. In intestinal epithelial cell lines, expression of IL-32 $\alpha$ mRNA and protein was enhanced by IL-1 $\beta$, interferon (IFN)- $\gamma$ and tumor necrosis factor (TNF)- $\alpha$. An inhibitor of phosphatidylinositol 3-kinase(LY294002) significantly suppressed the IL-1 $\beta$, IFN- $\beta \gamma$ and TNF- $\alpha$-induced IL-32 $\alpha$ mRNA expression, although MAPK inhibitors had no effect. Futhermore, LY294002 suppressed 
both IL- $1 \beta$-and TNF- $\alpha$-induced NF- $\mathrm{KB}$ activation, as well as IL- $1 \beta$-and TNF- $\alpha$-and IFN- $\gamma$-induced AP- 1 activation.

Conclusions: Epithelial IL-32 $\alpha$ expression was increased in IBD patients, and in particular CD patients. IL-32 might be involved in the pathophysiology of IBD as a proinflammatory cytokine. IL-32 $\alpha$ induction was dependent on interactions between the PI3K/Aktpathway and the NF- $\mathrm{B}$ /AP-1 system.

\section{4 \\ Plasmid IL-10 Enema Therapy for Mice with DSS Colitis}

\begin{tabular}{|c|}
\hline 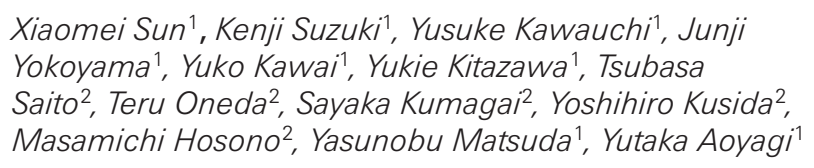 \\
\hline $\begin{array}{l}\text { 1Division of Gastroenterology and Hepatology, Niigata } \\
\text { University Graduate School of Medical and Dental } \\
\text { Sciences, Niigata, Japan, } 2 \text { Department of Life Science, } \\
\text { Niigata University Graduate School of Science and } \\
\text { Technology, Niigata, Japan }\end{array}$ \\
\hline
\end{tabular}

Aim: Interleukin-10 attracted attention as an immunoregulation/ anti-inflammation-related cytokine for curative effect in patients with inflammatory bowel disease (IBD), however, systemic administration of IL-10 brings severe side effects. This study examined the effect of local administration of IL-10 plasmid by enema for mice with chronic Dextran sodium sulfate (DSS) colitis.

Methods: Colitis was induced in 9 week-old C57BL/6 (B6) mice by giving drinking water with $3 \%$ DSS ad labium for 5 days. IL-10 gene expression plasmid or control plasmid was administered by enema on day 2 , and day 4 after induction of colitis. Mice were sacrificed on day 6 . The disease activity index (DAI) was calculated to evaluate the clinical course of colitis. Histological analysis by H-E staining, and immunohistochemistry with anti-Ki67 antibody were done.

Results: DAI was significantly reduced, and pathological lesions were attenuated in mice with DSS colitis by plasmid-IL-10 enema. IL-10 gene transfer inhibited the shortening of colon of mice with DSS colitis as compared with control vectors. The number of Ki67positive colonic epithelial cells was significantly increased in colitis mice by plasmid-IL-10 enema.

Conclusion: Plasmid-IL-10 enema ameliorated DSS colitis through anti-inflammatory and mild regenerative effects, suggesting that IL-10 gene transfer by enema could be an adjunctive therapeutic approach for IBD.
15

\section{The Role of MCP-1 Dependent Intestinal Macrophage Subset in Gut Homeostasis}

\author{
Yasuhiro Takada', Tadakazu Hisamatsu', Nobuhiko \\ Kamada1, Riko Saito', Tetsuro Takayama', Hitoshi \\ Ichikawa1, Taku Kobayashi', Hiroshi Chinen², Susumu \\ Okamoto ${ }^{1}$, Toshifumi Hibi ${ }^{1}$ \\ 'Division of Gastroenterology and Hepatology, Department \\ of Internal Medicine, Keio University School of Medicine, \\ Tokyo, Japan, ${ }^{2}$ University of the Ryukyus, Okinawa, Japan
}

Background and Aim: Intestinal macrophages (M $\varphi s)$ in normal intestine show anti-inflammatory phenotypes, and play a central role in regulation of immune responses against commensal bacteria. Indeed, intestinal $\mathrm{M} \varphi s$ express several anti-inflammatory molecules, including IL-10. Monocyte chemoattractant protein-1 (MCP-1) is a chemokine, which plays important role for monocytes or $\mathrm{M} \varphi$ s recruitment to the inflamed tissue. In the present study, we demonstrated that lamina propria macrophages in wild-type mice spontaneously produce large amount of MCP-1 in steady state and tried to clarify the role of MCP-1 dependent intestinal macrophage subset in gut homeostasis.

Methods: 1) Lamina propria macrophages (LPM $\varphi s)\left(\mathrm{CD}_{11} \mathrm{~b}^{+}\right.$ cells) were isolated from wild-type (WT) and MCP-1 deficient (MCP$\left.1^{-/-}\right)$mice using magnetic cell sorting. Isolated LPM $\varphi$ s were stained with mAbs F4/80, CD11c, CD11b or their isotype control Abs and analyzed using a FACSCalibur. Isolated LPM $\varphi$ s were then stimulated with commensal bacteria (Enterococcus faecalis) heat-killed antigens, and produced cytokines were assessed by cytometric beads array. 2) Colitis was induced in both WT and MCP-1/- mice by intake of dextran sulfate sodium (DSS) and analyzed the role of MCP-1.

Results: 1) MCP-1 dependent intestinal macrophage subset $\left(\mathrm{SSC}^{\mathrm{lo}} \mathrm{CD} 11 \mathrm{~b}^{+} \mathrm{F} 4 / 80^{+} \mathrm{CD} 11 \mathrm{c}^{-}\right)$was reduced in $\mathrm{MCP}-1^{-/-}$mice lamina propria and IL-10 production from CD11 ${ }^{+}$LPM $\varphi$ s (whole M $\varphi s$ ) in $\mathrm{MCP}-1^{-/}$mice was significantly reduced compare with WT mice. 2) DSS-induced colitis was exacerbated in MCP-1/- mice than WT mice. 3) Recruitment of MCP-1 dependent LPM $\varphi s$ was disturbed in inflamed mucosa of $\mathrm{MCP}-1^{-/-}$mice.

Conclusions: We first identified that intestinal macrophages produce constantly MCP-1 and MCP-1 dependent intestinal macrophages may recruit to suppress excess immune response and contribute gut homeostasis by producing IL-10.

16

\section{Colon Cancer Progression through IL-8-Induced EGF Signaling}

\author{
Satoshi Tanida, Makoto Sasaki, Hiromi Kataoka, \\ Takeshi Kamiya, Takashi Joh \\ Department of Gastroenterology and Metabolism, Nagoya \\ City University, Graduate School of Medical Sciences, \\ Nagoya, Japan
}

Background and Purpose: IL-8 induces colon cancer growth. We previously reported that IL-8, G-protein coupled receptor (GPCR) agonist, promoted cell proliferation through EGFR transactivation. 
After the ectodomain of proHB-EGF was cleaved by a disintegrin and metalloproteinase (ADAM) which was activated by GPCR agonist, C-terminal fragments of HB-EGF (HB-EGFc) were trafficked into nucleus. However, this mechanism by IL-8 is still unknown. We here investigated IL-8-induced trafficking of HB-EGFc as a new mechanism of colon cancer growth.

Method: HT-29 colon cancer cell line was employed. Cell proliferation was investigated by growth curve assay. Trafficking of HB-EGFc into nucleus was evaluated by immunofluorescence assay. Cell line stably expressing alkaline phosphatase-tagged HB-EGF was established to evaluate the ectodomain shedding of HB-EGF. KB-R7785, ADAM inhibitor, was used to block the shedding of HB-EGF and trafficking of HB-EGFc into nucleus. EGFR phosphorylation after IL-8 stimulation was examined by immunoprecipitation with anti-EGFR antibody followed by blotting with anti-phosphotyrosine antibody.

Results: IL- 8 induced cell proliferation, the shedding of HB-EGF and EGFR transactivation. IL-8 also promoted trafficking of HB-EGFc into nucleus and then PLZF, a gene repressor, was exported from nucleus to cytoplasma. KB-R7785 blocked IL-8-induced cell proliferation. That also blocked the shedding of HB-EGF, EGFR transactivation and trafficking of HB-EGFc into nucleus and nuclear export of PLZF.

Conclusions: IL- 8 induced trafficking of HB-EGFc into nucleus and nuclear export of PLZF to promote cell proliferation. Inhibition of HB-EGFc trafficking (HB-EGF shedding) may lead to new strategies for prevention of cell proliferation in colon cancer.

Key Word: IL-8, C-terminal fragments of HB-EGF, cell proliferation.

\section{7}

Synergistic Inhibitory Effect of Vitamin K2 and Interferon- $\beta$ on Angiogenesis of PIVKAII Producing Hepatocellular Carcinoma Cells

\author{
Kyoko Toda', Fumihiko Kaneko 2,3, Naoki Kumagai², Satoshi \\ Tsunematsu², Haruko Sugino ${ }^{4}$, Yukio Suzuki², \\ Kanji Tsuchimoto ${ }^{2}$, Hidetsugu Saito ${ }^{5}$, Toshifumi Hibi ${ }^{5}$ \\ ${ }^{1}$ Research center, ${ }^{2}$ Department of Internal Medicine, \\ Kitasato Instituite Hospital, ${ }^{3}$ Department of Internal \\ Medicine, Kitasato Medical Center Hospital, ${ }^{4}$ School of \\ Pharmaceutical Science, Kitasato University, ${ }^{5}$ Department \\ of Internal Medicine, School of Medicine, Keio University, \\ Japan
}

Background/Aims: We previously reported that combination of Vitamin K2 (VK2) and interferon (IFN)- $\beta$ significantly inhibited tumor growth and induced apoptosis of Protein induced by Vitamin $\mathrm{K}$ absence or antagonists (PIVKA)-II producing hepatocellular carcinoma (HCC) cell lines. Several reports have shown that angiogenesis is involved in early stage of carcinogenesis. Therefore, the aim of this study was to elucidate the combined effect of VK2 and IFN- $\beta$ on angiogenesis of HCC cell lines.

Methods: The in vitro angiogenesis induced by PIVKA-II was assessed as formation of tubules (capillary-like structures) of human umbilical vascular endothelial cells (HUVEC) co-cultured with diploid fibroblasts and examined the inhibitory effect of VK2 and IFN- $\beta$. Then we examined angiogenic effect of culture-supernatant of human
HCC (PLC/PRF/5, HepG2, SK-Hep-1) in this system, with or without VK2 and IFN- $\beta$.

Results: PIVKA-II augmented tubular formation of HUVEC in a dose-dependent manner as well as vascular endothelial growth factor (VEGF). VK2 and IFN- $\beta$ markedly suppressed the tubular formation in concert. Culture supernatant of PLC/PRF/5 and HepG2, which produced PIVKA-II and VEGF, also enhanced tubular formation of HUVEC. PIVKA-II and VEGF production of both cells were significantly reduced by treatment with VK2 and IFN- $\beta$. Neovascularization induced by the culture supernatant of both cells was significantly suppressed by combination treatment with VK2 and IFN- $\beta$ as compared with each alone.

Conclusions: We have shown that the combination treatment of VK2 and IFN- $\beta$ exerted synergistic inhibitory effect on the angiogenesis of PIVKA-II producing HCC. This combination regimen may represent potential new strategy against $\mathrm{HCC}$ in the future.

18

\section{Restoration of Adaptive Immunity in Innate Immunity Distorted HBV Carrier Model Mice by Antigen Pulsed Dendritic Cells}

Osamu Yoshida, Masanori Abe, S.M. Fazle Akbar, Teruki Miyake, Maho Hamada, Morikazu Onji

Department of Gastroenterology and Metabology, Ehime University Graduate School of Medicine, Japan

Background/Aims: Induction and maintenance of proper and effective immune responses against HBV is one of main purposes of immune therapy against chronic HBV infection. We showed that antigen-pulsed dendritic cells (DC) are capable of inducing HBVspecific adaptive immunity in HBV transgenic mice (HBV-Tg), an animal model of HBV carrier state. However, innate immunity is also distorted in chronic hepatitis B patients. To develop insights about effective regimen of immune therapeutic approaches in condition of distorted innate immunity, we produced natural killer (NK)-depleted HBV-Tg, characterized their innate immune status, and challenged them to induce HBV-specific adaptive immunity.

Methods: NK cells were depleted from mice by administration of anti-asialo GM1 antibody. Hepatitis B surface antigen (HBsAg)pulsed DC or HB vaccine (containing $\mathrm{HBsAg}$ ) was injected into NK-depleted HBV-Tg. We checked production of innate production, titer of anti-HBs in the sera and HBsAg specific cellular immune responses after administrating antigen-pulsed DCs or only vaccine.

Results: Spleen cell culture of HBsAg-pulsed DC injected HBV-Tg produced significantly higher amounts of inflammatory cytokines compared to those immunized with $\mathrm{HB}$ vaccine. Either small amounts or no anti-HBs were detected from NK-depleted HBV-Tg injected with $\mathrm{HB}$ vaccine. But all NK-depleted HBV-Tg injected with HBsAg pulsed DC exhibited anti-HBs in the sera. Also, HBsAgspecific proliferation of lymphocytes was seen in only HBsAg pulsed DC injected, but, not in HB vaccine-injected NK-depleted HBV-Tg.

Conclusion: This study explored the need of innate immunity for induction of adaptive immunity; the principle of which may be used for development better immune therapeutic regimen against cancers and chronic viral infections. 
Abe, M. 18

Adachi, S. 5

Andoh, A. 13

Aoyagi, Y. 14

Arakawa, T. 3, 9

Asakura, H. 12

Chinen, H. 15

Ebinuma, H. 10

Fazle Akbar, S.M. 18

Fujiwara, Y. 3, 9

Fujiyama, Y. 13

Fukata, N. 7

Funaki, J. 11

Hamada, M. 18

Handa, O. 2, 5, 11

Harada, K. 1

Hibi, T. $10,15,17$

Hirata, I. 2

Hirata, N. 3

Hisamatsu, T. 15

Hokari, R. 6

Hosomi, S. 3

Hosono, M. 12, 14

Ichikawa, H. 15

Inatomi, O. 13

Inoue, H. 4

Ishikawa, T. 5, 11

Joh, T. 16

Kaimul Ahsan, M. 8

Kajikawa, H. 2
Kamada, N. 15

Kamata, N. 3

Kamiya, T. 16

Kaneko, F. 17

Kataoka, H. 16

Kawaguchi, A. 6

Kawai, Y. 14

Kawauchi, Y. 12, 14

Kitazawa, Y. 14

Kobayashi, T. 15

Kokura, S. 2, 5, 11

Komoto, S. 6

Kono, M. 3

Koyabu, M. 7

Kumagai, N. 17

Kumagai, S. 14

Kurihara, C. 6

Kushida, Y. 12

Kusida, Y. 14

Masutani, H. 8

Matsuda, Y. 14

Matsunaga, H. 6

Matsushita, M. 7

Mishima, K. 4

Miura, S. 6

Miyake, T. 18

Miyoshi, H. 7

Mizushima, K. 2

Nagafuchi, S. 12

Nagao, S. 6

Naito, Y. 2, 5, 11

Nakamura, H. 8

Nakamura, M. 6

Nakane, K. 11
Nakanuma, Y. 1

Nishida, A. 13

Obara, K. 4

Ogawa, A. 13

Ohno, T. 11

Ojiro, K. 10

Okada, Y. 6

Okamoto, S. 15

Okayama, T. 5, 11

Okazaki, H. 9

Okazaki, K. 7

Okudaira, K. 6

Okuyama, H. 8

Omatsu, T. 2

Oneda, T. 14

Onji, M. 18

Oshitani, N. 3, 9

Otani, K. 9

Saito, H. 10, 17

Saito, I. 4

Saito, R. 15

Saito, T. 12, 14

Sasaki, M. 16

Sato, Y. 1

Shioya, M. 13

Sogawa, M. 3

Suekane, T. 3

Sugino, H. 17

Sun, X. 12, 14

Suzuki, K. 12, 14

Suzuki, Y. 17

Tada, S. 10

Takada, Y. 15
Takagi, T. 2, 5, 11

Takaoka, M. 7

Takayama, T. 15

Takebayashi, K. 6

Takeda, T. 11

Tanida, S. 16

Tanigawa, T. 9

Taniguchi, T. 7

Toda, K. 17

Tominaga, K. 3, 9

Tsuchimoto, K. 17

Tsujikawa, T. 13

Tsunematsu, S. 17

Tsuzuki, Y. 6

Uchida, K. 7

Wakabayashi, K. 10

Watanabe, C. 6

Watanabe, K. 3, 9

Watanabe, T. 3, 9

Yagi, Y. 13

Yamagami, H. 3, 9

Yamagishi, Y. 10

Yamamoto, S. 4

Yazumi, S. 7

Yodoi, J. 8

Yokoyama, J. 14

Yoshida, N. 2

Yoshida, O. 18

Yoshikawa, T. 2, 5, 11

\section{KARGER}

(C) 2008 S. Karger AG, Basel

Fax +4161306 1234

E-Mail karger@karger.ch

www.karger.com 